\title{
The optical properties of the G89.0+4.7 (HB 21) supernova remnant
}

\author{
F. Mavromatakis ${ }^{1}$, E. M. Xilouris ${ }^{2}$, and P. Boumis ${ }^{2}$ \\ 1 Technological Education Institute of Crete, General Department of Applied Scienses, PO Box 1939, 71004 Heraklion, \\ Crete, Greece \\ e-mail: fotis@physics.uoc.gr \\ 2 Institute of Astronomy \& Astrophysics, National Observatory of Athens, I. Metaxa \& V. Pavlou, P. Penteli, 15236 Athens, Greece
}

Received 29 December 2005 / Accepted 25 August 2006

\section{ABSTRACT}

\begin{abstract}
Flux-calibrated CCD images of HB 21 are presented, along with flux calibrated spectra of moderate resolution. The low ionization images reveal filamentary structures in the east, while the emission in the central to west areas may be either filamentary or patchy. The filaments in the east are correlated very well with the radio emission, but a smaller degree of correlation exists for the patchy emission. An area in the west exhibits strong sulfur emission and is probably associated to the remnant. Uncatalogued H II regions are present in the area of the remnant, mainly in the south. We find that an ellipse extending for $116^{\prime} \times 122^{\prime}$ incorporates most of the radio and optical emission. The actual physical extent of the remnant depends on the distance, which is not well determined since it ranges from 0.8 to $1.7 \mathrm{kpc}$. Deep long-slit spectra were also acquired at a number of locations. The H $\alpha$ emission is generally weak, typically below $15 \times 10^{-17} \mathrm{erg} \mathrm{s}^{-1} \mathrm{~cm}^{-2} \operatorname{arcsec}^{-2}$. The $\mathrm{H} \beta$ emission was not always detected, suggesting substantial attenuation of the light through the interstellar medium. The medium ionization line of [O III]5007 $\AA$ was not detected indicating low shock velocities into the interstellar clouds whose density is estimated to be a few atoms per $\mathrm{cm}^{3}$.
\end{abstract}

Key words. ISM: general - ISM: supernova remnants - ISM: individual objects: G 89.0+4.7

\section{Introduction}

The supernova remnant $\mathrm{HB} 21$ was discovered more than 50 years ago in the radio band (Brown \& Hazard 1953). Current estimates of the distance to the source differ significantly ranging from $0.8 \mathrm{kpc}$ to $1.7 \mathrm{kpc}$ (e.g. Tatematsu et al. 1990; Byun et al. 2006). A number of observations at different frequencies suggest a spectral index of $\sim 0.4$, clearly identifying the non-thermal nature of the radio emission (e.g. Reich et al. 2003, and references therein). Strong radio emission is located in the inner areas of the remnant, while filamentary emission is found at specific locations along its periphery (e.g. Uyaniker et al. 2003). The $856 \mathrm{MHz}$ image presented by Reich et al. (2003) reveals a well-defined boundary at an angular resolution of 14.5 .

Molecular, atomic, and radio continuum observations led Tatematsu et al. (1990) to propose the interaction of the remnant with the surrounding material, mainly along its eastern boundary. The atomic gas displays a smoother distribution compared to the clumpy nature of the $\mathrm{CO}$ emission (their Fig. 6a). It is believed that some of the detected clumps belong to the Cyg OB7 association. Koo et al. (2001) performed higher resolution molecular observations of the eastern half of the remnant. In the northern and southern areas the detected $\mathrm{CO}$ broad emission lines were attributed to fast-moving clumps overtaken by the blast wave. They also analyzed IRAS HIRES images to show that the infrared emission is due to shocks propagating into them.

HB 21 was originally detected in the $0.2-4.0 \mathrm{keV}$ band by the Einstein satellite (Leahy 1987) and was also detected in the soft X-ray band by ROSAT (Leahy \& Aschenbach 1996) during the All Sky survey. It has been proposed that this object belongs to the class of mixed morphology remnants due to its centrally brightened X-ray emission and shell like radio morphology (Pannuti et al. 2006). Subsequent observations of the brightest emission regions were performed by the ASCA satellite (Lee et al. 2001). The spectral fits point to the thermal nature of the X-ray emission with temperatures around $0.6 \mathrm{keV}$ and hydrogen column densities in the range of $2-4 \times 10^{21} \mathrm{~cm}^{-2}$. Similar temperatures $(0.6-0.7 \mathrm{keV})$ and hydrogen column densities have been obtained by Pannuti et al. (2006).

Even though this supernova is well-observed in radio wavelengths, less data are available on its optical morphological and spectral properties. Images in the $\mathrm{H} \alpha$ and [O III] lines were acquired by Lozinskaya (1972) but were not shown. Interferometric observations in the $\mathrm{H} \alpha$ line by Lozinskaya (1972, 1975, 1980) lead to the determination of an expanding shell velocity of $\sim 25 \mathrm{~km} \mathrm{~s}^{-1}$, while the highest observed velocities reached $60-80 \mathrm{~km} \mathrm{~s}^{-1}$.

Willis (1973) presented an optical image of HB 21 taken from deep red plates. However, the quality of the reproduced photograph does not permit a detailed analysis, and the author commented that the radio and optical features correspond poorly. $\mathrm{He}$ also suggested that absorption increases from the west towards the east parts of the remnant. However, van den Bergh (1978) did not detect any optical nebulosity in deep $\mathrm{H} \alpha$ and [S II] plate images, related to HB 21, obtained with the $1.2 \mathrm{~m}$ Schmidt telescope at Palomar mountain.

In this work, we present flux-calibrated CCD images of this remnant in the emission lines of $\mathrm{H} \alpha+[\mathrm{N} \mathrm{II}]$ and [S II]. Deep long-slit spectra were also acquired in a number of selected locations. In Sect. 2 information about the observations and the data analysis is provided, while in Sect. 3 we report on the results of the imaging and spectral observations. In Sect. 4, we discuss the 
Table 1. Journal of the observations.

\begin{tabular}{lll}
\hline \hline Filter & Date (UT) & $\begin{array}{l}\text { Total exp. time } \\
\text { (No of frames }^{b} \text { ) }\end{array}$ \\
\hline $\mathrm{H} \alpha+[\mathrm{N} \mathrm{II}]$ & $31 / 07 / 03$ & $2400 \mathrm{NE} \mathrm{(1)}$ \\
$\mathrm{H} \alpha+[\mathrm{N} \mathrm{II}]$ & $31 / 07 / 03$ & $4800 \mathrm{SE} \mathrm{(2)}$ \\
$\mathrm{H} \alpha+[\mathrm{N} \mathrm{II}]$ & $01 / 08 / 03$ & $4800 \mathrm{SW}(2)$ \\
$\mathrm{H} \alpha+[\mathrm{N} \mathrm{II}]$ & $01 / 08 / 03$ & $4800 \mathrm{NW}(2)$ \\
$\mathrm{H} \alpha+[\mathrm{N} \mathrm{II}]$ & $02 / 08 / 03$ & $4800 \mathrm{NE} \mathrm{(2)}$ \\
\hline$[\mathrm{S} \mathrm{II}]$ & $31 / 07 / 03$ & $4800 \mathrm{NE} \mathrm{(2)}$ \\
{$[\mathrm{S} \mathrm{II}]$} & $31 / 07 / 03$ & $4800 \mathrm{SE} \mathrm{(2)}$ \\
{$[\mathrm{S} \mathrm{II}]$} & $01 / 08 / 03$ & $4800 \mathrm{SW}(2)$ \\
{$[\mathrm{S} \mathrm{II}]$} & $01 / 08 / 03$ & $4800 \mathrm{NW}(2)$ \\
{$[\mathrm{S} \mathrm{II}]$} & $02 / 08 / 03$ & $4800 \mathrm{SE} \mathrm{(2)}$ \\
{$[\mathrm{S} \mathrm{II}]$} & $02 / 08 / 03$ & $2400 \mathrm{NE} \mathrm{(1)}$ \\
\hline Cont red & $31 / 07-02 / 08 / 03$ & $4320(18)$ \\
\hline
\end{tabular}

${ }^{a}$ Total exposure times in s. ${ }^{b}$ Number of individual frames.

optically-related properties of this remnant, and summarize the results of the current in Sect. 5.

\section{Optical observations}

The $0.3 \mathrm{~m}$ Schmidt-Cassegrain telescope at Skinakas Observatory, Crete, Greece, was used for the imaging observations. Multiple pointings, typically two per field, were performed from July 31 to August 02,2003 in order to map this extended remnant. A $1024 \times 1024$ Thomson CCD was used resulting in a $70^{\prime} \times 70^{\prime}$ field of view and an image scale of $4^{\prime \prime}$ per pixel. A detailed log of the observations is given in Table 1. The peak transmittances of the $\mathrm{H} \alpha+[\mathrm{N} \mathrm{II}]$ and [S II] filters occur at 6560 and $6708 \AA$, and their FWHM are 75 and $20 \AA$, respectively. All data frames were projected to an ra-dec grid in J2000 coordinates with the aid of the HST Guide star catalogue (Lasker et al. 1999).

Standard IRAF ${ }^{1}$ and MIDAS $^{2}$ routines were utilized to reduce the data. The data were bias subtracted and flat-field corrected using a series of well exposed twilight flat-fields. The spectrophotometric standard stars HR 5501, HR 7596, HR 7950, HR 8634, HR 9087, and HR 718 were observed in order to provide absolute flux calibration of the data (Hamuy et al. 1992, 1994).

The long-slit spectra were acquired with the $1.3 \mathrm{~m}$ RitcheyCretien telescope at Skinakas Observatory from July 30 to August 02, 2003. The spectral log (Table 2) lists the coordinates of the slit centers, the number of available spectra at each location, and the total exposure time of each spectrum. The data were taken with a 1300 line $\mathrm{mm}^{-1}$ grating and a $800 \times 2000 \mathrm{SITe}$ CCD allowing the range of $4750 \AA-6815 \AA$ to be explored. The angular width of the slit is 7'.7 (east-west direction), and its length extends for 7.9 in the south-north direction. The current setup results in a resolution of $\sim 7$ and $\sim 11 \AA$ in the red and blue wavelengths, respectively. The spectrophotometric standard stars HR 5501, HR 7596, HR 9087, HR 718, and HR 7950 were observed for the spectral calibration.

\footnotetext{
1 The Image Reduction and Analysis Facility is a general purpose software system available from http://iraf. noao.edu

2 The Munich Image and Data Analysis System is an image processing and data reduction software system available from http://www.eso.org/projects/esomidas
}

Table 2. Spectral log.

\begin{tabular}{llll}
\hline \hline Slit centers & & Area & Total exp. time $^{a}$ \\
\hline$\alpha$ & $\delta$ & & (No of spectra $^{b}$ ) \\
$20^{\mathrm{h}} 50^{\mathrm{m}} 12^{\mathrm{s}}$ & $51^{\circ} 20^{\prime} 58^{\prime \prime}$ & I & $7200(2)$ \\
$20^{\mathrm{h}} 51^{\mathrm{m}} 04^{\mathrm{s}}$ & $50^{\circ} 46^{\prime} 32^{\prime \prime}$ & II & $7200(2)$ \\
$20^{\mathrm{h}} 45^{\mathrm{m}} 20^{\mathrm{s}}$ & $50^{\circ} 04^{\prime} 32^{\prime \prime}$ & III & $3600(1)$ \\
$20^{\mathrm{h}} 43^{\mathrm{m}} 21^{\mathrm{s}}$ & $49^{\circ} 27^{\prime} 55^{\prime \prime}$ & IV & $7200(2)$ \\
$20^{\mathrm{h}} 39^{\mathrm{m}} 39^{\mathrm{s}}$ & $50^{\circ} 03^{\prime} 28^{\prime \prime}$ & V & $3600(1)$ \\
$20^{\mathrm{h}} 43^{\mathrm{m}} 58^{\mathrm{s}}$ & $50^{\circ} 51^{\prime} 04^{\prime \prime}$ & VI & $7200(2)$ \\
$20^{\mathrm{h}} 41^{\mathrm{m}} 43^{\mathrm{s}}$ & $51^{\circ} 00^{\prime} 50^{\prime \prime}$ & VII & $3600(1)$ \\
\hline
\end{tabular}

${ }^{a}$ Number of spectra obtained. ${ }^{b}$ Total exposure times in $\mathrm{s}$.

\section{The optical emission}

The images in the $\mathrm{H} \alpha+[\mathrm{N} \mathrm{II}]$ and [S II] filters of the area of HB 21 are presented in Figs. 1 and 2, respectively. The field appears somewhat complex due to the presence of a significant number of diffuse emission structures. Some of these patches are found outside the radio emission boundaries of HB 21 in the south-west and are thus not related to the remnant. However, weaker and diffuse emission patches are located in its central, western areas (Fig. 1). A search in the SIMBAD database did not reveal any known bright nebulae. The closest recorded bright nebula is LBN 381 (Lynds 1965), which is located at a declination of $48^{\circ} 30^{\prime}$. Generally, $\mathrm{H}$ II regions display [S II]/H $\alpha$ ratio of less than 0.35 , typically around 0.2 (e.g. Hunter 1992), while in supernova remnants this ratio exceeds the value of 0.4 and is usually greater than 0.5 (e.g. Smith et al. 1993). Thus, areas of stronger sulfur emission might imply a possible association to the remnant. The overall diffuse emission, mainly present in the $\mathrm{H} \alpha+[\mathrm{N} \mathrm{II}]$ image, may not be related to the remnant given the significant attenuation of the emission in the [S II] image.

The low ionization images reveal three filamentary structures, two in the north east and one in the north west. The ones in the north east correlate well with filamentary radio emission, while the one in the north west also correlates with the radio emission in the area, although not with filamentary emission. It is interesting to note that the majority of the sulfur's patchy emission in the central areas of HB 21 displays some degree of correlation with the radio emission at $4850 \mathrm{MHz}$ (areas A, B, C; Fig. 3). Furthermore, the correlation with the $4850 \mathrm{MHz}$ radio emission (Condon et al. 1994) in area D is excellent and leads us to propose that this patchy emission is associated to the remnant. The correlation also extends to location VII, an area that emits shock-heated radiation (Sect. 3.2).

Moderate-resolution spectra were acquired at seven different locations around the area of HB 21 (Figs. 1 and 4). The measured fluxes are scaled to an $\mathrm{H} \alpha$ flux of 100 and are listed in Table 3. The quoted fluxes are the average of the individual fluxes weighted by their signal to noise in those cases where two spectra are available at the same location. The upper limits on the electron densities $(1 \sigma)$ are derived with the nebular package (Shaw and Dufour 1995) within the IRAF software.

The $\mathrm{H} \alpha$ fluxes are weak and are found in the range of $6-16 \times 10^{-17} \mathrm{erg} \mathrm{s}^{-1} \mathrm{~cm}^{-2} \operatorname{arcsec}^{-2}$. In some locations $\mathrm{H} \beta$ line emission is not detected, while our best-measured flux of this line in location " $\mathrm{V}$ " suggests moderate interstellar extinction $(\mathrm{H} \alpha / \mathrm{H} \beta=5.0 \pm 0.4)$. The $\mathrm{H} \alpha / \mathrm{H} \beta$ ratio cannot be accurately determined from the rest of the spectra. However, locations "IV" and "V" are outside the radio boundaries of the remnant and most likely are not related to it. Moreover, the low $\mathrm{H} \alpha / \mathrm{H} \beta$ ratios may suggest that these regions lie in the foreground. 


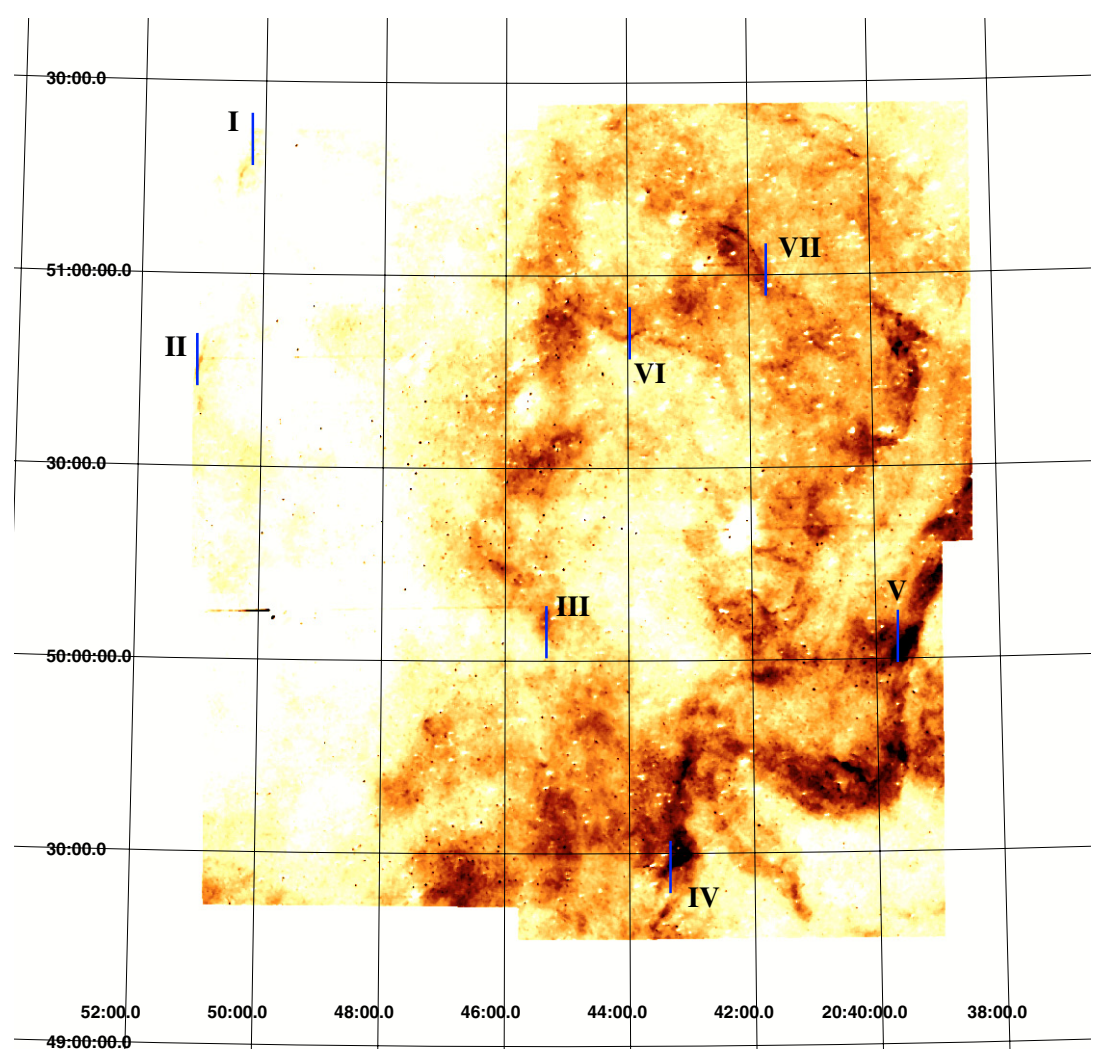

Fig. 1. The field of HB 21 in the $\mathrm{H} \alpha+[\mathrm{N} \mathrm{II}]$ filter. The shadings run linearly from 7 to $45 \times 10^{-17} \mathrm{erg} \mathrm{s}^{-1} \mathrm{~cm}^{-2} \operatorname{arcsec}^{-2}$. The long rectangles mark the locations where long-slit spectra were obtained. The image has been smoothed to suppress the residuals from the imperfect continuum subtraction.

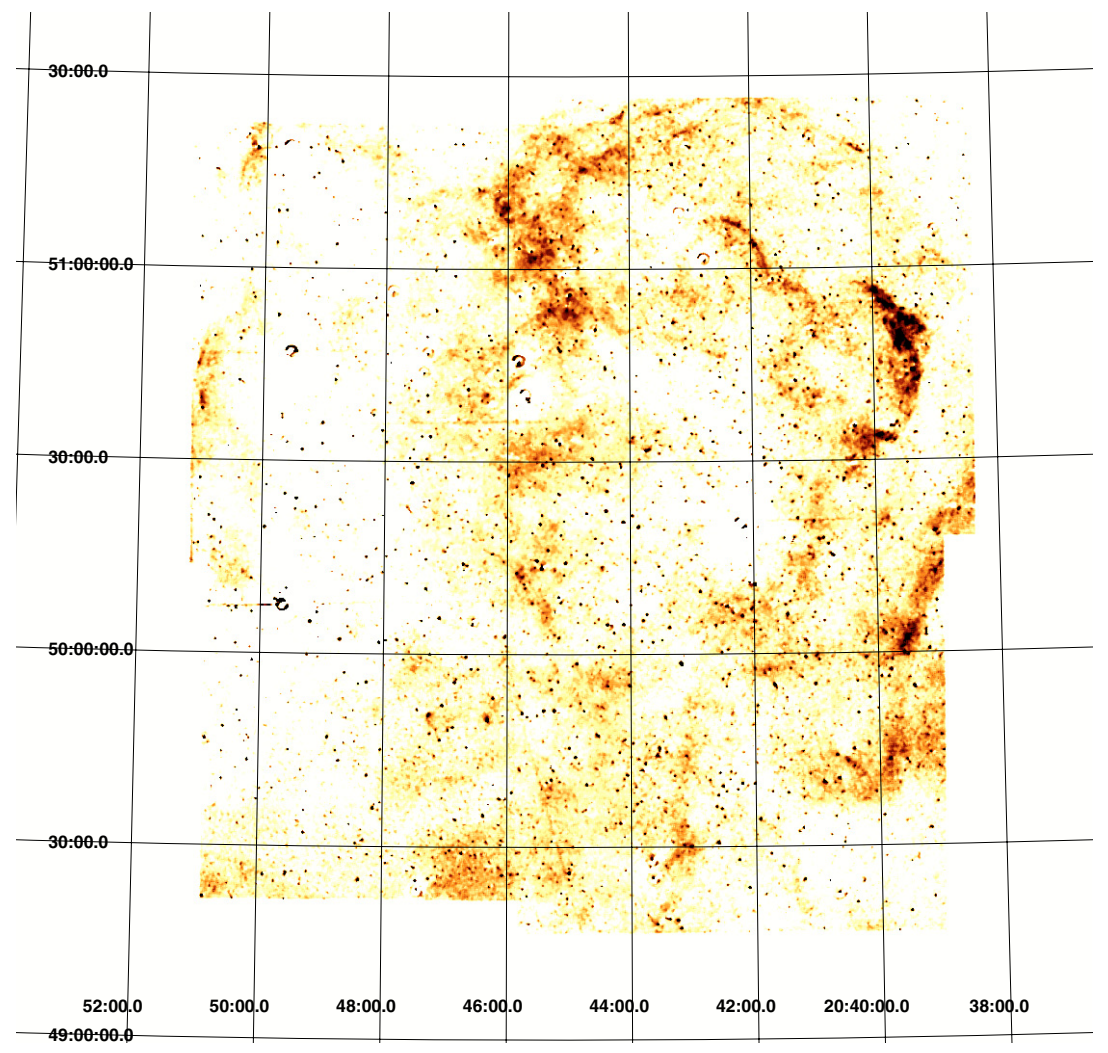

Fig. 2. Areas of strong sulfur emission [S II] are good candidates for an association with the remnant because the sulfur filter suppresses the background emission, as well as the emission from HII regions. The image has been smoothed to suppress the residuals from the imperfect continuum subtraction, while the shadings run linearly from 1 to $9 \times 10^{-17} \mathrm{erg} \mathrm{s}^{-1} \mathrm{~cm}^{-2} \operatorname{arcsec}^{-2}$. 


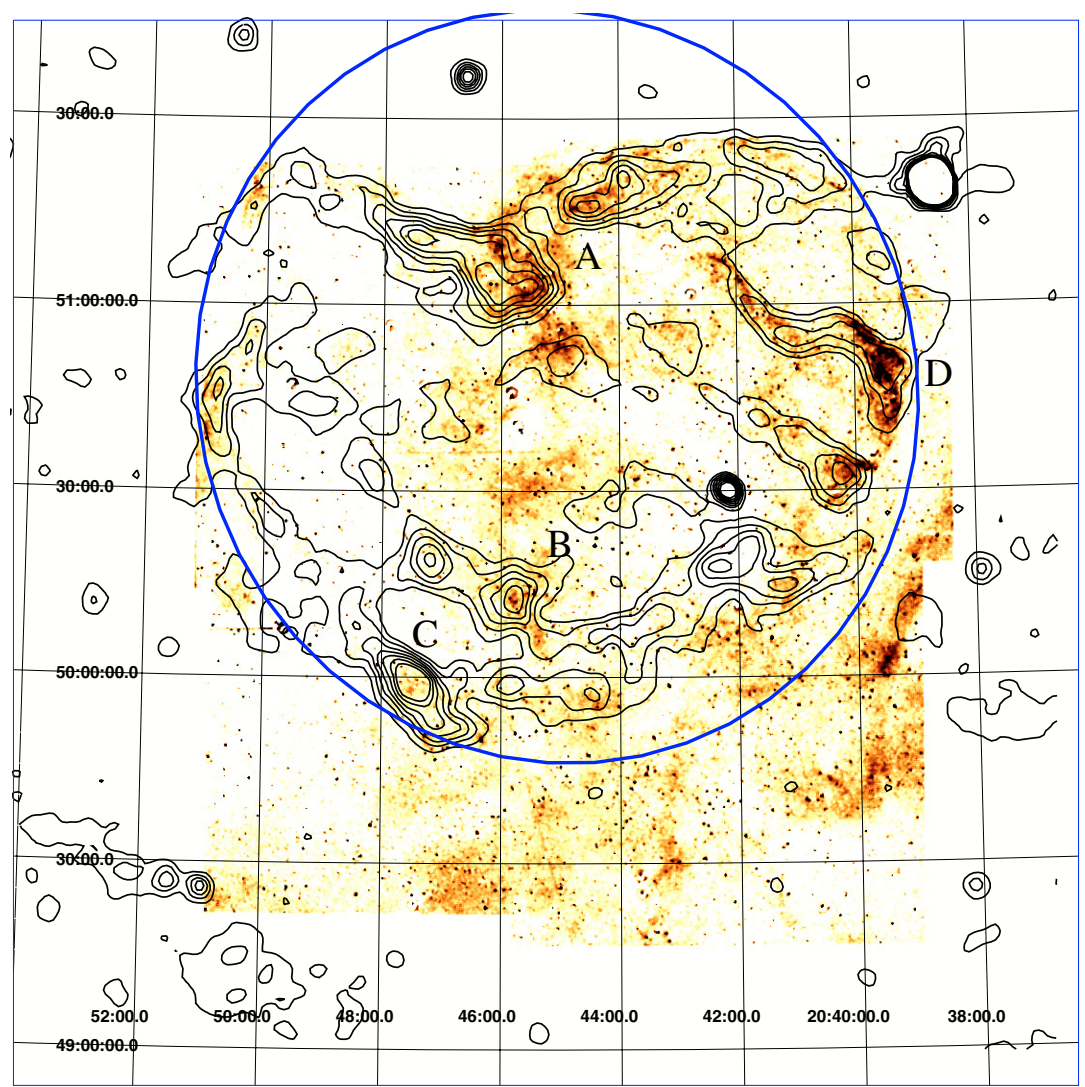

Fig. 3. The field of HB 21 in the [S II] filter (background image) with the $4850 \mathrm{MHz}$ contours overlaid. The contours scale linearly, every $0.022 \mathrm{Jy} /$ beam, from 0.01 to $0.14 \mathrm{Jy} /$ beam (Condon et al. 1994). The letters A to D refer to areas of emission that may be associated to the remnant, while the ellipse drawn encompasses the majority of the radio and optical emission.

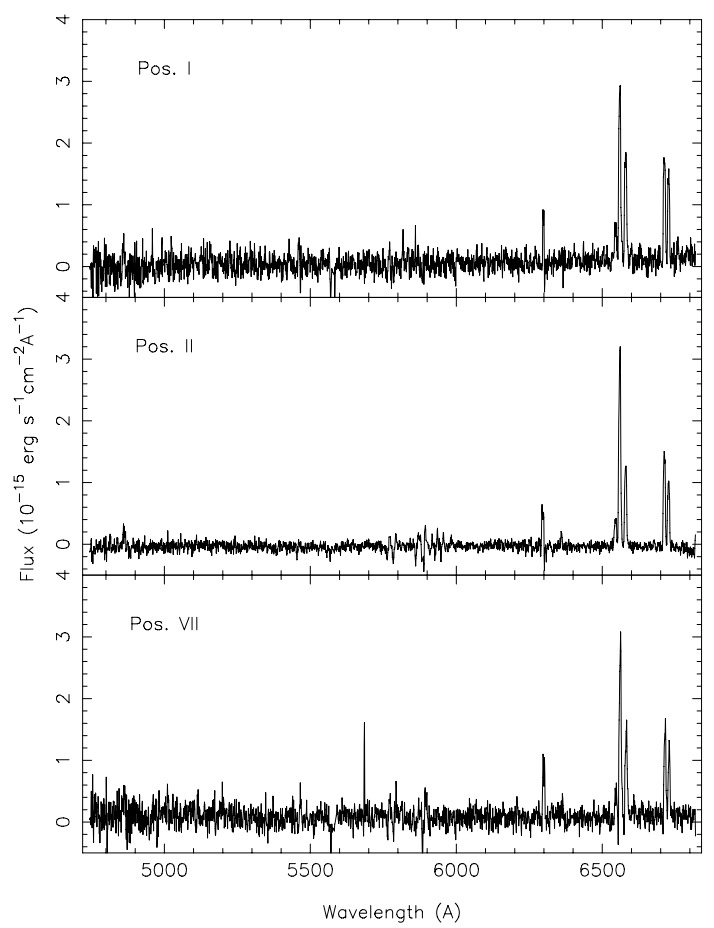

Fig. 4. Typical long-slit spectra from locations I, II, and VII in the area of the remnant HB 21. More details can be found in Sect. 3.2 and Table 3.

The average sulfur line ratio is 1.35 over the area of the remnant, indicative of low electron densities. Given the relatively low significance of the measured fluxes, the one-sigma upper limits on the electron densities are quoted in Table 3 . The wide range of electron densities does not allow us to draw secure conclusions about the shock velocity and/or the strength of the magnetic field. The measured [S II] $/ \mathrm{H} \alpha$ ratios exceed 0.53 , with the exception of locations IV and V, suggesting the presence of shock-heated gas.

The medium ionization line of oxygen at $5007 \AA$ is not detected in our spectra pointing to low shock velocities and possibly to a neutral cloudy medium. Emission from neutral oxygen at $6300 \AA$ is detected in the majority of the measured spectra but is a factor of 2-3 less than the [N II] 6548, $6584 \AA$ flux. These two observational data suggest that slow shocks $\left(<100 \mathrm{~km} \mathrm{~s}^{-1}\right)$ propagate into the interstellar clouds.

\section{Discussion}

Flux-calibrated images and spectra of the extended remnant HB 21 are presented for the first time. Even though this remnant is well observed in radio wavelengths, little information is available about its optical properties, with the exception of its kinematic behavior (Lozinskaya 1980, and references therein).

The low-ionization images reveal filamentary emission in the east and patchy emission in the central to west areas of the remnant. The optical filaments in the east display a high degree of correlation with the radio emission, while the patchy emission shows a reduced degree of correlation. It is possible that the diffuse-patchy emission covering an extended area is not related to the remnant since such emission is quite suppressed in the [S II] image. The detection of strong sulfur emission in the 
Table 3. Relative line fluxes.

\begin{tabular}{llllllll}
\hline \hline & I & II & III & IV & V & VI & VII \\
\hline Line $(\AA)$ & F & F & F & F & F & F & F \\
$4861 \mathrm{H} \beta$ & - & $9(3)$ & $15(4)$ & - & $20(8)$ & $18(5)$ & - \\
$6300[\mathrm{O}$ I] & $17(6)$ & $17(9)$ & $9(3)$ & - & - & - & $30(6)$ \\
$6548[\mathrm{~N} \mathrm{II}]$ & $20(7)$ & $15(6)$ & $15(6)$ & $14(5)$ & $14(7)$ & $13(6)$ & $16(3)$ \\
$6563 \mathrm{H} \alpha$ & $100(33)$ & $100(46)$ & $100(37)$ & $100(33)$ & $100(49)$ & $100(43)$ & $100(19)$ \\
$6584[\mathrm{~N} \mathrm{II}]$ & $65(21)$ & $44(20)$ & $44(16)$ & $50(17)$ & $46(23)$ & $53(22)$ & $52(10)$ \\
$6716[\mathrm{~S} \mathrm{II}]$ & $59(20)$ & $48(23)$ & $38(15)$ & $27(9)$ & $26(13)$ & $31(12)$ & $45(9)$ \\
$6731[\mathrm{~S} \mathrm{II}]$ & $48(17)$ & $34(16)$ & $26(11)$ & $20(7)$ & $20(10)$ & $22(9)$ & $34(7)$ \\
\hline $\mathrm{Absolute} \mathrm{H} \alpha$ flux & 6.5 & 12.8 & 15.5 & 10.5 & 13.5 & 6.5 & 5.7 \\
\hline $\mathrm{H} \alpha / \mathrm{H} \beta$ & - & $11(3)$ & $7(4)$ & - & $5.0(8)$ & $5.5(5)$ & - \\
{$[\mathrm{S} \mathrm{II}] / \mathrm{H} \alpha$} & $1.08(20)$ & $0.83(24)$ & $0.64(16)$ & $0.47(11)$ & $0.46(16)$ & $0.53(14)$ & $0.80(10)$ \\
$\mathrm{I}(6716) / \mathrm{I}(6731)$ & $1.23(13)$ & $1.41(13)$ & $1.45(9)$ & $1.37(6)$ & $1.30(8)$ & $1.36(7)$ & $1.31(6)$ \\
$\mathrm{n}_{\mathrm{e}}{ }^{2}$ & $<380 \mathrm{~cm}^{-3}$ & $<120 \mathrm{~cm}^{-3}$ & $<130 \mathrm{~cm}^{-3}$ & $<320 \mathrm{~cm}^{-3}$ & $<330 \mathrm{~cm}^{-3}$ & $<290 \mathrm{~cm}^{-3}$ & $<410 \mathrm{~cm}^{-3}$ \\
\hline
\end{tabular}

${ }^{1}$ In units of $10^{-17} \mathrm{erg} \mathrm{s}^{-1} \mathrm{~cm}^{-2} \operatorname{arcsec}^{-2}$.

$21 \sigma$ upper limits.

All fluxes are not corrected for interstellar extinction Numbers in parentheses represent the signal-to-noise ratio of the quoted values All fluxes normalized to $\mathrm{F}(\mathrm{H} \alpha)=100$.

majority of the observed locations clearly suggests that this optical emission is associated to the remnant (Table 3). The morphology of the optical emission is either filamentary or patchy. The filamentary nature may be related to the viewing geometry, while the patchy appearance may be attributed to the effects of turbulent magnetic fields (e.g. Raymond \& Curiel 1995).

Flux-calibrating the $\mathrm{H} \alpha+[\mathrm{N} \mathrm{II}]$ and [S II] images can identify areas of emission which may be associated to the remnant. Emission in the areas A, B, C, and D may be associated to the remnant given their spatial correlation with the radio emission (Fig. 3). We also show the low-ionization images as an RG composite to allow for an easier identification of any emission areas possibly related to the remnant (Fig. 6). The reddish areas of emission indicate areas prominent in the [S II] emission. The correlation in area B is further supported by the characteristics of the long-slit spectrum at location III. A promising candidate is the emission detected in area D (Fig. 3). The sulfur emission in this area is, at least, a factor of 2 stronger than in other areas where the long-slit spectra clearly identify the shock-heated nature of the detected emission. Furthermore, the sulfur emission is still strong, even when compared to the corresponding $\mathrm{H} \alpha+[\mathrm{N} \mathrm{II}]$ emission. Considering the $[\mathrm{S}$ II $] / \mathrm{H} \alpha+[\mathrm{N}$ II $]$ ratios measured in the low ionization images and the $[\mathrm{S} \mathrm{II}] / \mathrm{H} \alpha$ values from the spectra, we propose that the emission in area D is associated to the remnant. This suggestion is supported by radio data, at a frequency of $4850 \mathrm{MHz}$ and a resolution of $7^{\prime}$ available from the Green Bank survey (Condon et al. 1994). Despite this low resolution, the correlation between the radio and the optical emission is immediately evident both in area D and location VII.

To compare the [S II] optical emission from HB 21 with the infrared emission from this region, we made use of the IRAS resolution-enchanced (HiRes; Aumann et al. 1990) images of this area at $60 \mu \mathrm{m}$. The comparison is shown in Fig. 5 with the [S II] emission on the background and the $60 \mu \mathrm{m}$ emission presented with contours. It shows that the infrared emission quite nicely traces most of the structures observed in the north, as well as in the south. In their analysis, Koo et al. (2001) argue that the enchanced far-infrared color and the morphological correlation between the infrared and the $\mathrm{CO}$ emission suggest that the infrared emission is caused by shocks propagating into the clouds. The correlation between the optical and the infrared emission that we find supports this argument.
The failure to detect oxygen emission at $5007 \AA$ indicates low shock velocities around or below $100 \mathrm{~km} \mathrm{~s}^{-1}$, as Cox \& Raymond (1985) have shown in their work. Using the relation of Fesen \& Kirshner (1980),

$n_{[\mathrm{SII}]}=45 n_{\mathrm{c}} V_{\mathrm{s}, 100}^{2}$

with $n_{\text {[SII }]}<120 \mathrm{~cm}^{-3}$ (upper limit from location II), and a shock velocity of $\sim 100 \mathrm{~km} \mathrm{~s}^{-1}\left(V_{\mathrm{s}, 100}=1\right)$, results in cloud densities $n_{\mathrm{c}}$ around $2.5 \mathrm{~cm}^{-3}$ or higher, depending on the actual shock velocity. For example, for a shock velocity of $80 \mathrm{~km} \mathrm{~s}^{-1}$, the cloud density would increase to $\sim 4 \mathrm{~cm}^{-3}$. We note here that these densities are reasonable for the ISM inhomogeneities that are responsible for the optical emission (Raymond et al. 1988).

McKee \& Cowie (1975) obtained the relation

$\mathrm{E} 51=2 \times 10^{-5} \beta^{-1} n_{\mathrm{c}} V_{\mathrm{s}, 100}^{2} r_{\mathrm{s}}^{3}$,

which can be used as a rough estimate of the explosion energy. The variable $\beta$ is on the order of $1-2$, while E51 is the explosion energy in units of $10^{51} \mathrm{erg}$ and $\mathrm{r}_{\mathrm{s}}$ the shock radius in pc. Applying the upper limit of $\sim 120 \mathrm{~cm}^{-3}$ on the electron density (location II), along with Eq. (1) and for a typical radius of $14 D_{0.8 \mathrm{kpc}} \mathrm{pc}$, we obtain an upper limit on E 51 of $0.1 D_{0.8 \mathrm{kpc}}^{3}$. This is a factor of 10 less than the energy usually assumed in a supernova explosion. However, we also consider the new distance estimate of $1.7 \mathrm{kpc}$ proposed by Byun et al. (2006). In this case, the upper limit on the explosion energy becomes $\sim 1$. The absence of a widely accepted distance to HB 21 allows a wide range of possible explosion energies. We note here that Lozinskaya $(1975,1980)$ quotes an energy in the range of $2-5 \times 10^{49} \mathrm{erg}$ for a distance of $1 \mathrm{kpc}$. Lazendic and Slane (2006), in a recent study of three supernova remnants including HB 21, use the $0.8 \mathrm{kpc}$ distance and estimate an explosion energy of $\sim 10^{49}$ erg for the Sedov-Taylor case of expansion and an energy in the range of $10^{50}-10^{51}$ erg in the case of the evaporating cloud model of White \& Long (1991).

HB 21 is an interesting object for further studies because it appears to be evolved, and it exhibits filamentary optical emission in the east and also in the west, while radio and X-ray emission are detected as well. Optical kinematic data at the positions of the detected filaments would allow the cloud velocities to be determined and compared with those estimated from X-ray 


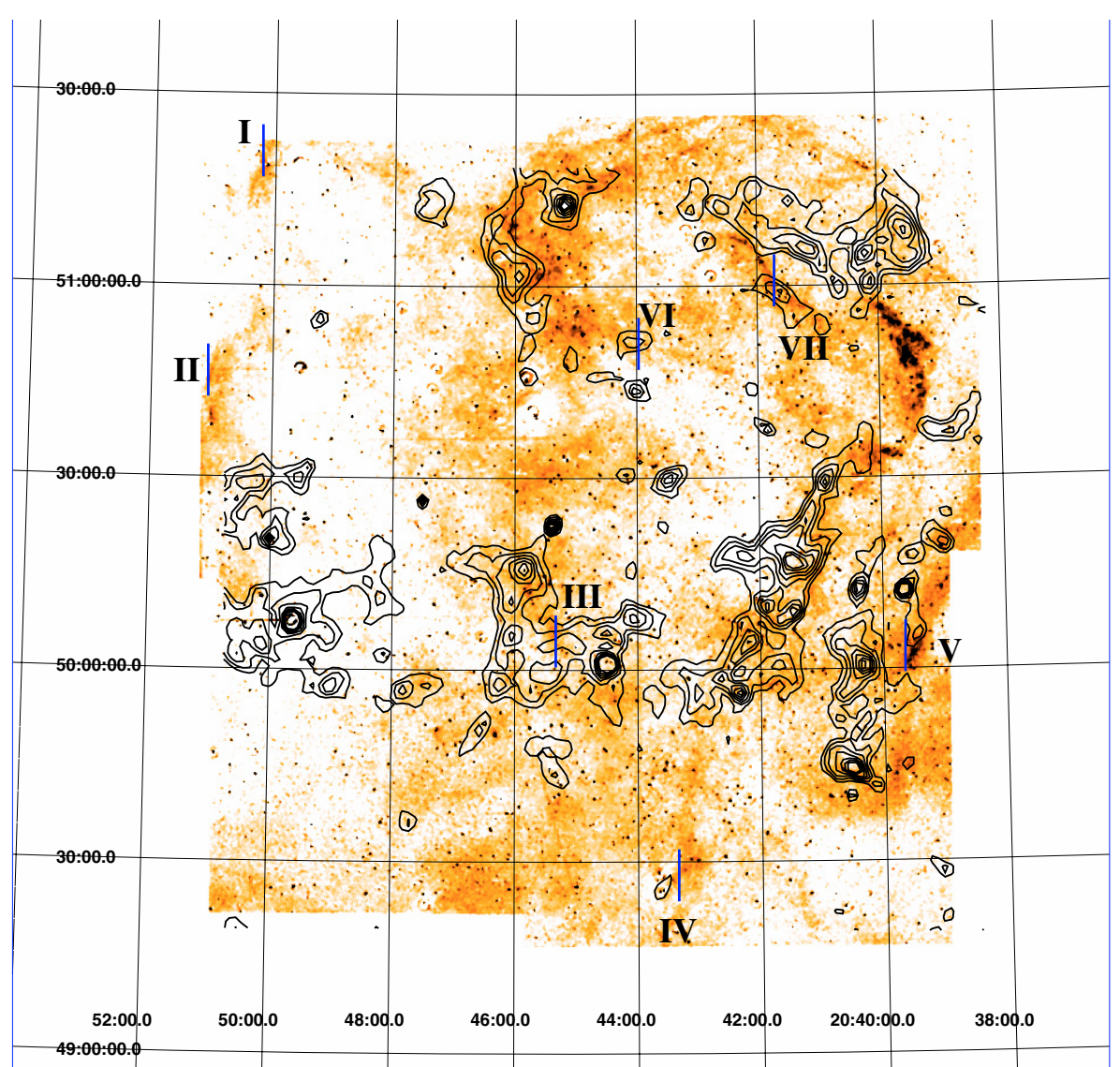

Fig. 5. The field of HB 21 in the [S II] filter (background image) with the $60 \mu \mathrm{m}$ IRAS/HiRes contours overlaid. The contours show the emission from 2 to $10 \mathrm{MJy} / \mathrm{sr}$ on 10 levels. The slit projections on the sky are shown for comparison purposes.

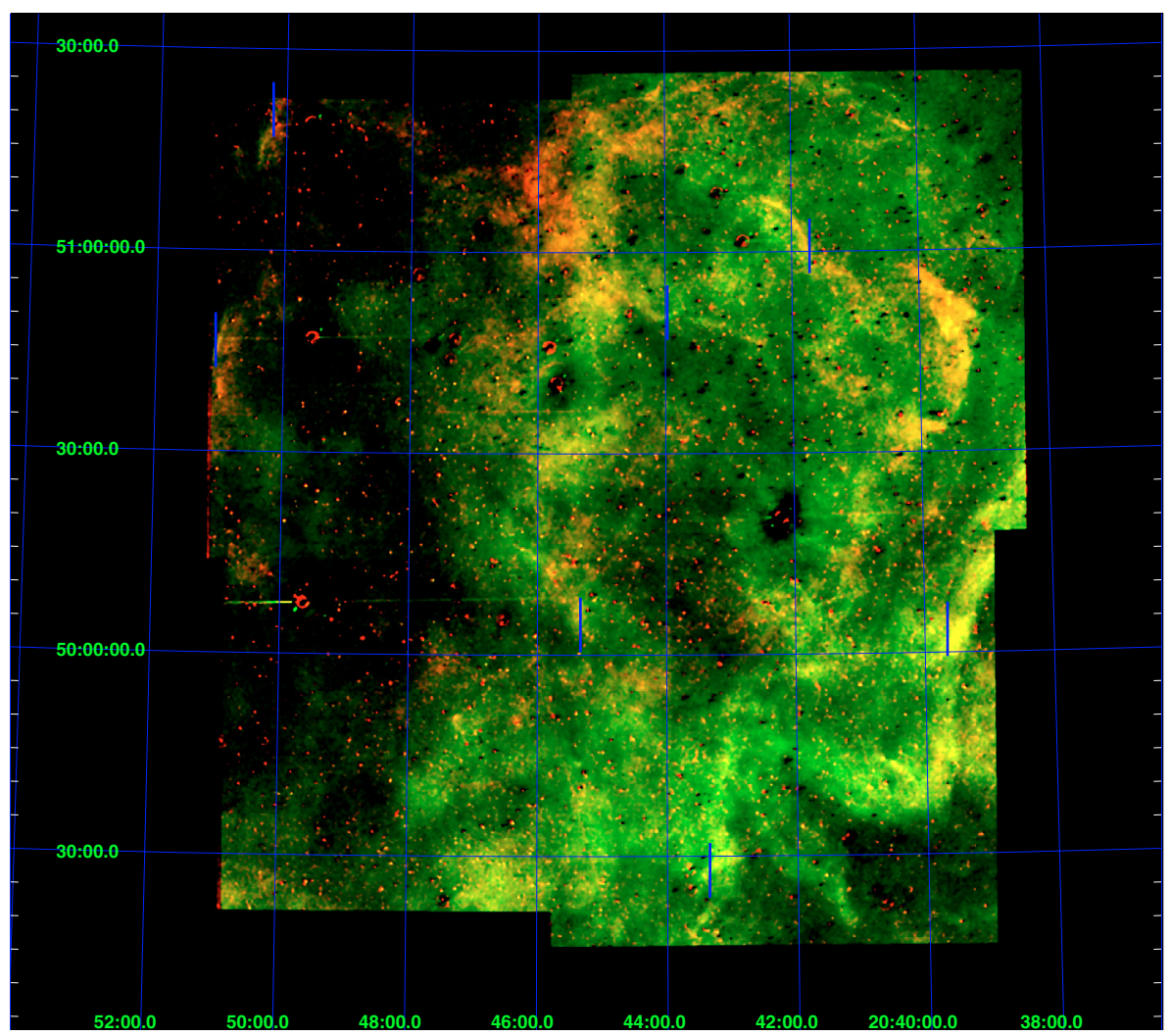

Fig. 6. This figure shows an RG composite based on the $\mathrm{H} \alpha+[\mathrm{N} \mathrm{II}]$ (greenish) and [S II] (reddish) low ionization images. The majority of the diffuse emission appears to emit mainly in the $\mathrm{H} \alpha$ and [N II] emission lines. The slit projections on the sky are also shown for comparison purposes. 
data and those estimated from models. Our knowledge for remnants beyond the adiabatic phase of their evolution could then be advanced.

\section{Conclusions}

The extended supernova remnant HB 21 was observed for the first time with deep CCD images and spectra. Filamentary and patchy structures were detected that appear correlated with the radio emission. The flux-calibrated images indicate that the $\mathrm{H} \alpha$, [N II], and [S II] emission is weak. Deep long-slit spectra also suggest significant attenuation of the optical emission by the interstellar gas. The $\mathrm{H} \beta$ line emission is either non-detectable or weakly present. The strength of the oxygen line at $5007 \AA$ points to slow shock propagating into the interstellar clouds. The density of these clouds is estimated to be a few particles per cubic centimeter.

Acknowledgements. The authors would like to thank the referee for comments and suggestions that significantly improved this work. Skinakas Observatory is a collaborative project of the University of Crete, the Foundation for Research and Technology-Hellas, and the Max-Planck-Institut für Extraterrestrische Physik. This research made use of data obtained through the High Energy Astrophysics Science Archive Research Center Online Service, provided by the NASA/Goddard Space Flight Center.

\section{References}

Aumann, H. H., Fowler, J. W., \& Melnyk, M. 1990, AJ, 99, 1674 Brown, R. H., \& Hazard, C. 1953, MNRAS, 113, 123

Byun, D. Y., Koo, B. C., Tatematsu, K., \& Sanada K. 2006, ApJ, 637, 283
Cioffi, D. F., McKee, C. F., \& Bertschinger, E. 1988, ApJ, 334, 252

Condon, J. J., Broderick, J. J., Seielstad, G. A., Douglas, K., \& Gregory, P. C. 1994, AJ, 107, 1829

Cox, D. P., \& Raymond, J. C. 1985, ApJ, 298, 651

Fesen, R. A., \& Kirshner, R. P. 1980, ApJ, 242, 1023

Hamuy, M., Walker, A. R., Suntzeff, N. B., et al. 1992, PASP, 104, 533

Hamuy, M., Suntzeff, N. B., Heathcote, S. R., et al. 1994, PASP, 106, 566

Hunter, D. A. 1992, ApJS, 79, 469

Koo, B. C., Rho, J., Reach, W. T., Jung, J., \& Mangum, J. 2001, ApJ, 552, 175

Lasker, B. M., Russel, J. N., \& Jenkner, H. 1999, in the HST Guide Star Catalog, version 1.1-ACT, The Association of Universities for Research in Astronomy, Inc.

Lazendic, J. S., \& Slane, P. O. 2006, ApJ, 647, 350

Leahy, D. A. 1987, MNRAS, 228, 907

Leahy, D. A., \& Aschenbach, B. 1996, A\&A, 315, 260

Lee, H. G., Rho, J., Koo, B. C., Petre, R., \& Decourchelle, A. 2001, AAA 198th meeting, June 2001

Lynds, B. T. 1965, ApJS, 12, 163

Lozinskaya, T. A. 1972, SvA, 16, 219

Lozinskaya, T. A. 1975, SvA, 19, 21L

Lozinskaya, T. A. 1980, A\&A, 84, 26

McKee, C. F., \& Cowie, L. 1975, ApJ, 195, 715

Pannuti, T., Rho, J., Borkowski, K. J., \& Cameron, P. B. 2006, AJ, submitted Raymond, J. C., \& Curiel, S. 1995, The Fifth Mexico-Texas Conference on Astrophysics: Gaseous Nebulae and Star Formation, 3, 199

Raymond, J. C., Hester, J. J., Cox, D., et al. 1988, ApJ, 324, 869

Reich, W., Zhang, X., \& Fürst, E. 2003, A\&A, 408, 961

Shaw, R. A., \& Dufour, R. J. 1995, PASP, 107, 896

Smith, R. C., Kirshner, R. P., Blair, W. P., Long, K. S., \& Winkler, P. F. 1993, ApJ, 407, 564

Tatematsu, K., Fukui, Y., Landecker, T. L., \& Roger, R. S. 1990, A\&A, 237, 189

Uyaniker, B., Landecker, T. L., Gray, A. D., \& Kother, R. 2003, ApJ, 585, 785

van den Bergh 1978, ApJS, 38, 119

White, R. L., \& Long, K. S. 1991, ApJ, 373, 543

Willis, A. G. 1973, A\&A, 26, 237 